\title{
Caracterización del flujo peatonal en espacios de transición: Caso Estación Vía Argentina y Piex
}

\section{Characterization of the pedestrian flow in transition spaces: Case of Vía Argentina and Piex Station}

\author{
Mariadny Guerra ${ }^{1,2 *}$, Amilcar Pérez ${ }^{l}$, Sharon Arauz ${ }^{l}$, Ariel Arosemena ${ }^{l}$, Jorge Perén ${ }^{1,2}$ \\ ${ }^{1}$ Facultad de Arquitectura y Diseño, ${ }^{2}$ Sustainable Building and City Research Group - SusBCity, Ciudad de Panamá \\ Universidad de Panamá,
}

\begin{abstract}
Resumen La transitabilidad posee una relación directa con el entorno construido, logrando beneficiar o perjudicar la vitalidad del tejido urbano, donde el flujo de peatones representa su principal éxito, disminuyendo el uso de vehículos y encaminándonos a una ciudad sostenible. Este estudio busca un análisis cuantitativo y cualitativo del flujo de peatones a través de los espacios de transición, tomando como caso los predios de la estación Vía Argentina del Metro de Panamá en la cual se emplaza el edificio Piex; implementando una metodología directa, compuesta por dos tipos de mediciones: la medición objetiva (grabación) en dos horarios distintos (12:00 a 1:00 p. m. y 5:00 p. m. a 6:00 p. m.) y la subjetiva (encuesta). El mapeo del trayecto de los peatones en el área de estudio muestra patrones significativamente distintos entre cada uno de los dos horarios estudiados. En el horario de 12:00 p. m. a 1:00 p. m. muchos peatones usan las aceras próximas a los edificios mientras que en el horario de la tarde, de 5:00 p.m. a 6:00 p. m, emplean más la acera paralela a la Vía España. Tomando en cuenta que según los resultados de la encuesta realizada en el horario de 12:00m. a 1:00 p. m. los peatones prefieren transitar por el área techada producida por los edificios, y realizan una caminata utilitaria, ya que se ven motivados a trasladarse por estudios y trabajo.
\end{abstract}

Palabras clave Transitabilidad, entorno construido, comportamiento al caminar, auditoría de transitabilidad, morfología urbana.

\begin{abstract}
Walkability has a direct relationship to the built environment. It benefits or damages the urban factory's vitality, where achieving pedestrian flow means success by reducing cars usage and follows the path to a sustainable city. This research makes a quantitative and qualitative analysis of pedestrian flow in transition spaces using the areas surrounding Via Argentina Metro Station in Panama City, where the Piex building is found; by implementing a direct methodology, composed by two measurement types: objective measurement (recording) during two different times of the day (12:00 to 1:00p. m. and 5:00p. m. to 6:00p. m. ) and subjective measurement (survey). The pedestrian path mapping in the study area shows significantly different patterns between each of the two schedules studied. In the hours of 12:00 p. m. to 1:00 p. m., many pedestrians use the sidewalks next to the buildings while in the afternoon hours from 5:00 p. m. to 6:00 p. m., they use the sidewalk parallel to the España Avenue. Taking into account that according to the results of the survey conducted from 12:00 p. m. to 1:00 p. m., pedestrians prefer to walk through the roofed area produced by the buildings and make a utilitarian walk since they are motivated to move for studies and work.
\end{abstract}

Keywords Walkability, built environment, walking behavior, walkability audit, urban morphology.

* Corresponding author: marievol_21@hotmail.com

\section{Introducción}

El caminar es interpretado como un transporte verde, que tiene un bajo nivel de impacto ambiental [1], el cual representa una actividad física diaria para la ciudadanía. Al incentivar esta actividad se incrementa la sostenibilidad y se mejora la salud de la población [1], [2],[3]. Caminar según sus motivaciones se clasifica en caminata utilitaria y caminata recreativa [4]. La caminata utilitaria es la que tiene un destino; es decir se origina y termina en diferentes lugares (ejemplo: los viajes). Por el contrario, la caminata recreativa está definida por la ausencia del destino; es decir, se origina y termina en el mismo lugar [4].

Por otro lado, la transitabilidad es la medición del grado amigable que tiene un sector para con los peatones, se utiliza 
para analizar la relación entre los patrones al caminar (walking behavior) y el entorno construido junto con su impacto en la sostenibilidad de la ciudad [1]. De acuerdo con [5] los aspectos constantes en las diferentes mediciones de transitabilidad son: (a) Presencia de aceras. b) Características de acceso universal, direccionalidad de ruta y conectividad de red de calle. (c) Seguridad de los tratamientos de cruce a nivel. (d) Ausencia de tráfico pesado y de alta velocidad. (e) Separación de peatones (f) Uso de suelo. (h) Diversidad en la construcción. (i) Paisajismo. (j) Interés visual y sentido del lugar según lo definido en las condiciones locales. (k) Seguridad real o percibida. Estos aspectos se ven claramente reflejados en nuestra área de estudio.

El área de estudio se encuentra en los predios de la estación Vía Argentina del Metro de Panamá, próximo a la Vía España. En su configuración encontramos el edificio Piex, el cual cuenta con espacios de transición; áreas semiabiertas de encuentro, de estar, pasaje o conexiones urbanas con ventajas en la eficiencia energética y calidad ambiental [6].

La necesidad de espacios óptimos para la circulación libre de los usuarios, tanto vehicular como peatonal es un problema del diario vivir para el panameño. Esto trae consigo la deficiencia del transporte público, que, a su vez, consecuentemente incrementa la cantidad de vehículos. Finalmente, el usuario se ve en la necesidad de implementar el transporte peatonal, que es una adición mucho más reciente a los procesos de planificación y que aún se aborda con mucha menos intensidad, seriedad y financiación, en contraste con el gasto de miles de millones de dólares destinados a comprender y optimizar la planificación vehicular, ignorando su importante papel en la reducción de la congestión [5] para lograr un ahorro energético en la disminución del uso vehicular y por ende el consumo de petróleo, para impactar positivamente en la sostenibilidad de la ciudad.

\subsection{Objetivo}

El propósito de este estudio es el análisis cuantitativo y cualitativo del trayecto de peatones en las inmediaciones de edificios con espacios de transición, evaluando la transitabilidad dentro del entorno construido.

\section{Metodología}

Se realizó una revisión bibliográfica de artículos y documentos relacionados con el tema, para identificar los avances a nivel nacional e internacional. La metodología implementada en este estudio fue propuesta por Rafiemanzelat et al. (2016)[1], de la cual aplicamos solo el método directo, compuesto por la medición objetiva, correspondiente al uso de datos de observación llamado auditoría de transitabilidad (walkability audit) a través de video o grabaciones, para producir resultados cuantitativos a partir de los hechos; y la medición subjetiva a través de encuestas para obtener resultados cualitativos a partir de la percepción de los usuarios.

Luego de visitas al sitio y recorridos a lo largo del área de estudio, se logró identificar que en los usos de suelos predominaba el mixto urbano (figura. 1), representado por un variado grupo de comercios como panaderías, restaurantes, bancos, entre otros que complementan la vitalidad brindada por la estación Vía Argentina del Metro de Panamá.

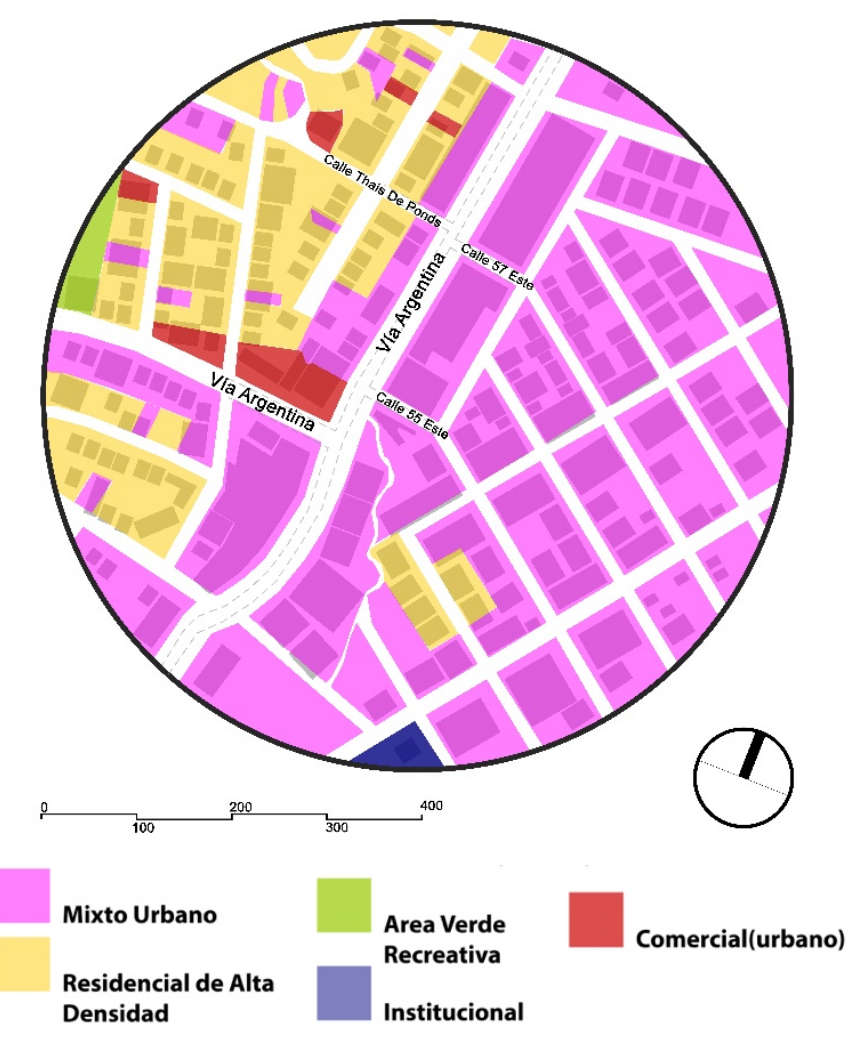

Figura 1. Mapa de usos de suelos.

\subsection{Medición subjetiva (encuesta)}

En la elaboración de la encuesta se tomó en cuenta la brevedad, así como también la simplicidad de las preguntas, con el objetivo de que fuera fácil y rápido de comprender para el encuestado, de manera a obtener la información esencial [7]. Estos criterios fueron importantes para tener una respuesta positiva por parte de los peatones, pues la mayoría transita con el tiempo limitado.

Con el objetivo de encuestar a la mayor cantidad de usuarios y obtener resultados más verídicos, la encuesta fue aplicada en los horarios de mayor flujo de peatones, tomando como referencia los locales comerciales próximos, cuyos horarios de apertura son alrededor de las 8:00 a. m. - 9:00 a.m. En un radio de 500 metros predominó la presencia de 
restaurantes dentro del uso mixto urbano, lo que nos sugirió establecer el horario de almuerzo (12:00 m. - 1:00 p. m.) para la encuesta.

Finalmente, la encuesta se realizó vía celular a través de un enlace proporcionado por una página web (onlineencuesta.com), que facilitó el proceso.

\subsection{Medición objetiva (video)}

La elaboración de los videos necesitó de cierto grado de evaluación para escoger los encuadres, priorizando los puntos de enfoque y los datos que se querían obtener. Por la extensión del área de estudio se necesitaron cuatro puntos de cámaras figura 2, en los cuales se utilizaron tres cámaras Canon EOS Rebel T6 con un lente de campo de visión 18-55mm, y una Go Pro-Hero con un campo de visión gran angular de 17.2 $\mathrm{mm}$.

La evaluación de los horarios de las grabaciones fue influenciada por el promedio de usuarios que utilizan la estación Vía Argentina proporcionado por el Metro de Panamá [8], de la cual utilizamos dos horarios: (a) 12:00 m. - 1:00 p. m., con un promedio de 2,564 usuarios y (b) 5:00 p. m. - 6:00 p. m., con el mayor promedio diario de 4,900 usuarios.
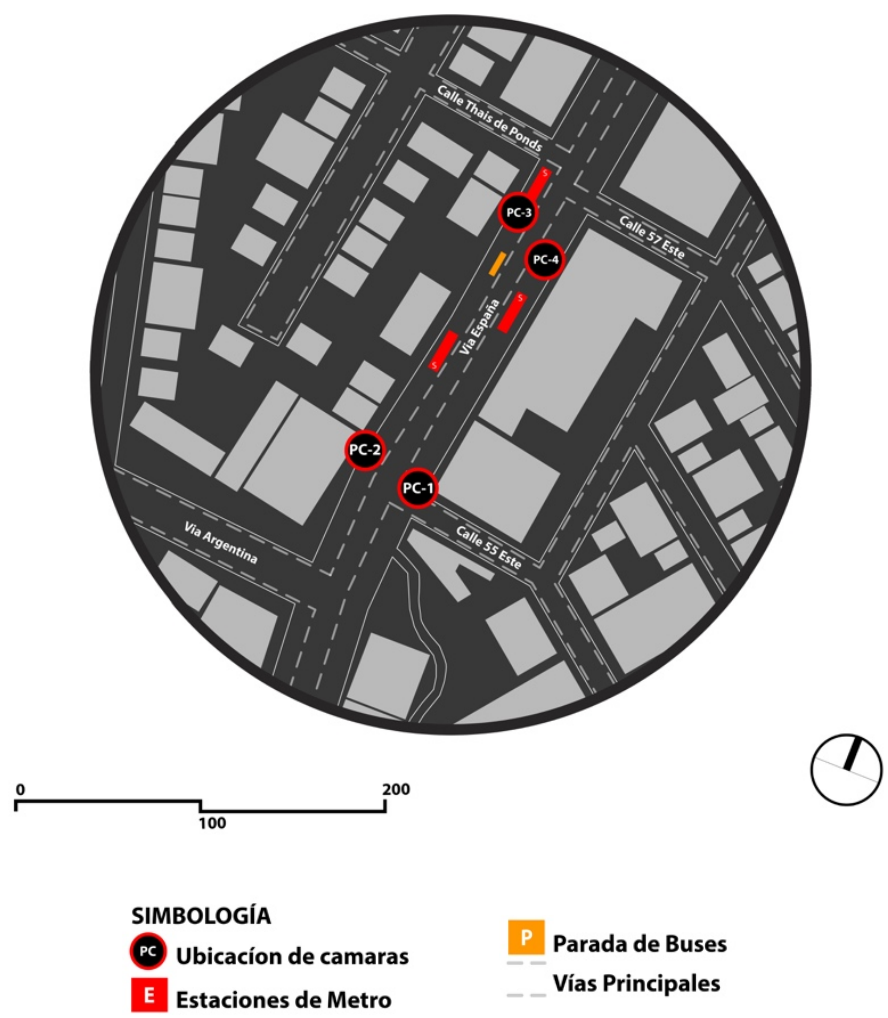

Parada de Buses Vías Principales

Figura 2. Mapa de ubicación de cámaras.

Se realizaron las dos grabaciones, con los mismos encuadres de cámaras, donde la PC-1 mira hacia el Edificio
Piex, la PC-2 hacia entrada del metro estación Vía Argentina diagonal al Subway de la Vía España, la PC-3 hacia la parada de buses atrás de la estación del metro - diagonal a la caja de ahorro de la Vía España, y la PC-4 ubicada diagonal a Melo Vía España mirando a la entrada de la estación Vía Argentina.

\subsection{Procesamiento de datos}

Se realizó la recopilación y organización de los datos, verificando que todo estuviera completo.

Los datos de la encuesta fueron obtenidos de una forma más rápida al implementar la Plataforma online "encuestas". Sin embargo, el procesamiento de los videos resultó más complejo al momento de iniciar los conteos por la cantidad de personas que transitaban. Este proceso nos llevó a realizar los mapas con la herramienta Adobe Ilustrador, la cual nos facilitó la modificación y edición de los gráficos.

\section{Resultados}

A través de las mediciones realizadas en el área de estudio se obtuvieron los siguientes datos, tomando en cuenta que no son conclusivos; ya que existen factores específicos que influenciaron y afectaron la determinación de los resultados a continuación.

\subsection{Medición subjetiva (encuesta)}

Se realizó una encuesta a algunos de los peatones que transitaron por el área de estudio, buscando información general y necesaria para el análisis de su comportamiento en los espacios de transición.

La cantidad de personas encuestadas en el horario de $12: 00$ m. a 1:00 p. m. fueron 40 y de estas un 62.10\% (25 personas) eran mujeres y el $37.90 \%$ (15 personas) eran hombres. La mayoría eran de contextura media y cargaban paquetes o libros con ellos. Ninguno de los peatones encuestados tenía algún problema de movilidad física y tampoco se entrevistó a ninguna mujer en estado de gestación.

La mayoría de los peatones encuestados eran mayores de edad, un 57\% oscila entre los 18 y 30 años. Solo un $24.14 \%$ eran extranjeros; cabe resaltar que algunos de estos se sentían inseguros de contestar la encuesta por no ser nacionales.

Un $82.76 \%$ de los transeúntes, viven lejos del área de estudio y la mayoría recorren el área para llegar a sus empleos y centros de estudio o universidades, figura 3. Un 3.4\% solamente realiza diligencias en el área. Un $44.83 \%$ pasa por el área de estudio diariamente y en su gran mayoría utilizan el Metro de Panamá, lo cual confirma que es un influyente clave. Muy pocas personas llegan a la zona sin un medio de transporte auxiliar, lo que responde a la distancia lejana en donde viven estos. 


\section{Motivo de su \\ desplazamiento}

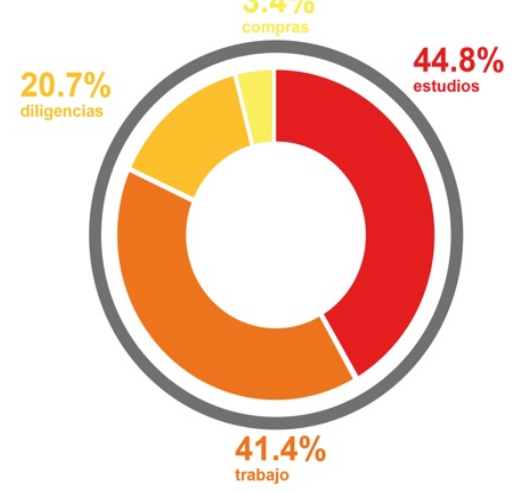

Figura 3. Gráfico de resultado: Motivo de desplazamiento.

Con respecto al espacio de transición, un $62.07 \%$ de los encuestados prefieren transitar por el área techada y un $37.93 \%$ prefieren la vereda descubierta, figura 4 . Cabe resaltar que esta encuesta se realizó a medio día y podemos afirmar que debido a la intensidad del sol, la mayoría de los peatones prefieren estar bajo la sombra, sin embargo, en el estudio realizado de 12:00 m. a 1:00 p. m., la gran parte de los peatones transitaban por las aceras próximas a la Vía España y las áreas descubiertas lo cual responde a la intención de realizar el análisis en dos horarios diferentes.

\section{ESPACIO DE TRANSICIÓN}

\section{¿Vereda abierta 0 vereda techada?}

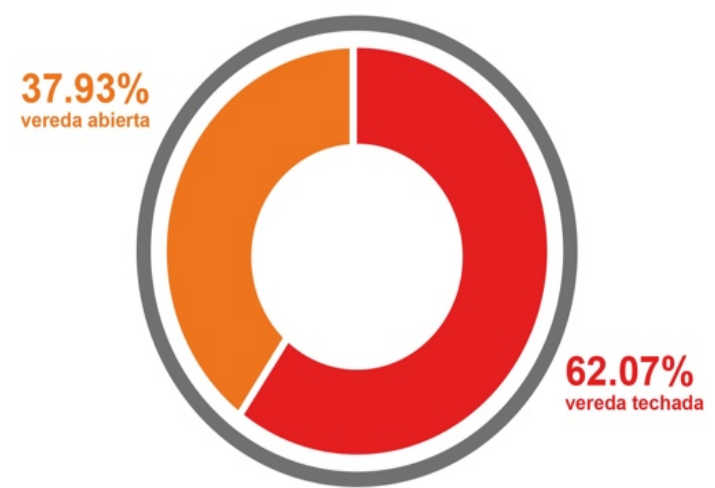

Figura 4. Gráfico de resultado: Preferencia dentro del espacio de transición, vereda abierta o techada de encuesta.

Se les solicitó a los peatones valorar el área con respecto a ciertas características: condiciones ambientales, seguridad peatonal e infraestructura. El resultado obtenido fue positivo y puede decirse que fue valorado en una media de 3.5 a 4.0 siendo el 5.0 el máximo valor a otorgar (excelente). Un $89.70 \%$ de los peatones se sienten seguros en el área estudiada y el $10.30 \%$ restante considera que hay inseguridad en el entorno.

\subsection{Medición objetiva (videos)}

3.2.1 Horario de 12:00 m. - 1:00 p. $\mathrm{m}$.

En este horario de 12:00 m. - 1:00 p. m. el día 4 de julio de 2018 , transitaron un promedio de 2470 personas

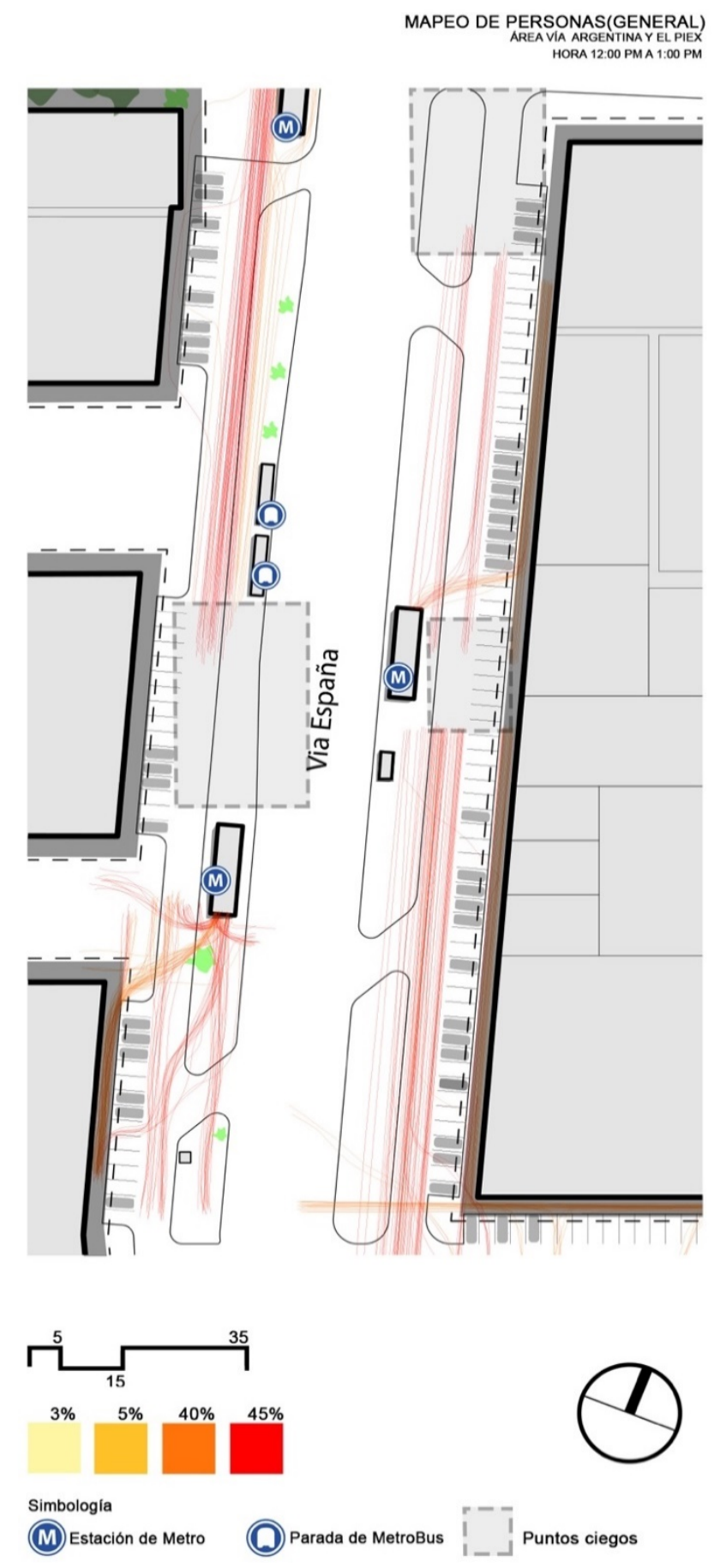

Figura 5. Gráfico General: Flujo de peatones, horario de 12:00 m. - 1:00 p.m. 
De este promedio el $30.52 \%$ (754 personas) por la $\mathrm{PC}-2$, el $27.48 \%$ (679 personas) por la $\mathrm{PC}-4$, el $25.22 \%$ (623 personas) se desplaza por la PC-1 (abajo del área techada del edificio Piex) figura 6 y el $16.76 \%$ (414 personas) por la PC-3. Lo que nos confirma que el mayor flujo de peatones se da próximo a las entradas del Metro, ya que este proporciona la principal conectividad con el resto de la ciudad.

Cabe destacar que en este horario estaba notablemente soleado, en la cual alrededor del $45 \%$ de los usuarios transitaron por el área abierta, y un $40 \%$ por el área techada.

Enfocándonos en la PC-1 donde se encuentra el edificio Piex con un espacio de transición techado, identificamos un total de 623 personas, donde el $70 \%$ transita por el área techada y un $5 \%$ al $15 \%$ por el área abierta figura 6 , lo que nos indica que el espacio de transición del Piex es funcional, ya que su área sombreada atrae a muchos peatones.

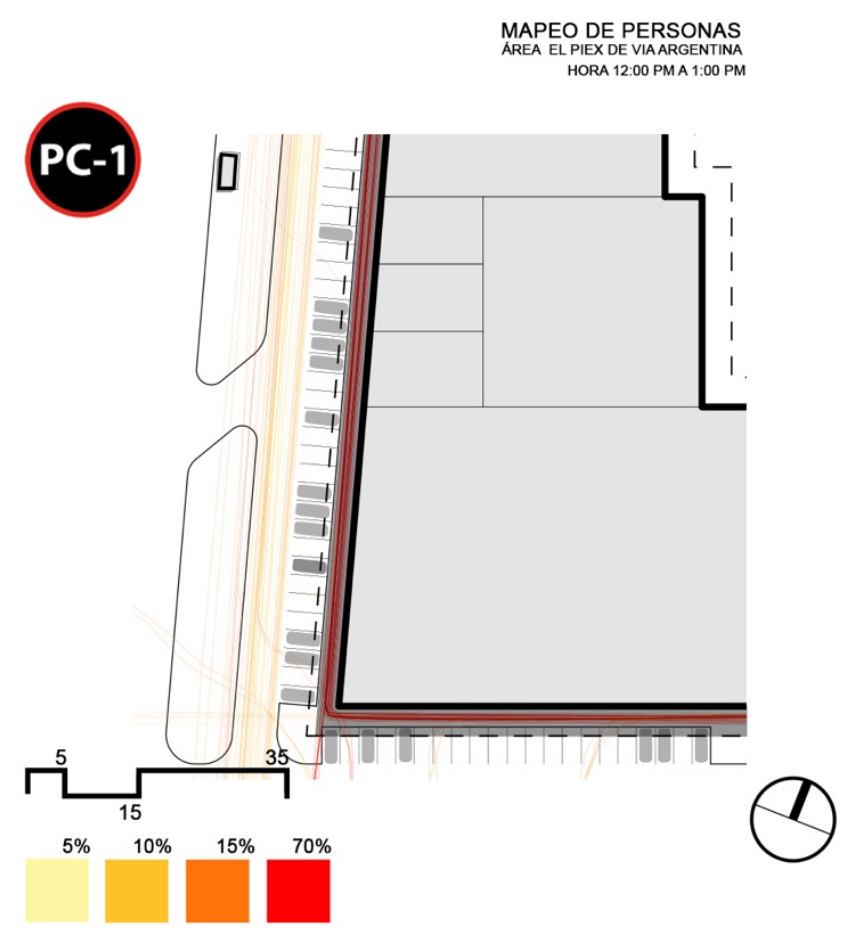

Figura 6. Gráfico de resultado: Ampliación del Piex, horario de 12:00 m. $-1: 00$ p. m.

\subsubsection{Horario de 5:00 p. m. - 6:00 p. $\mathrm{m}$.}

En este horario de 5:00 p.m. - 6:00 p. m. el día 06 de junio del 2018 transitaron un promedio de 4109 personas distribuidas a lo largo de la amplia área de estudio, figura 7.

De este promedio el 30.52\% (1254 personas) se desplazan por la PC-1 (edificio Piex) figura8, el 28.40\% (1167 personas) por la PC-4, 26.75\% (1099 personas) por la PC-2, y finalmente $14.33 \%$ (589 personas) por la PC-3.

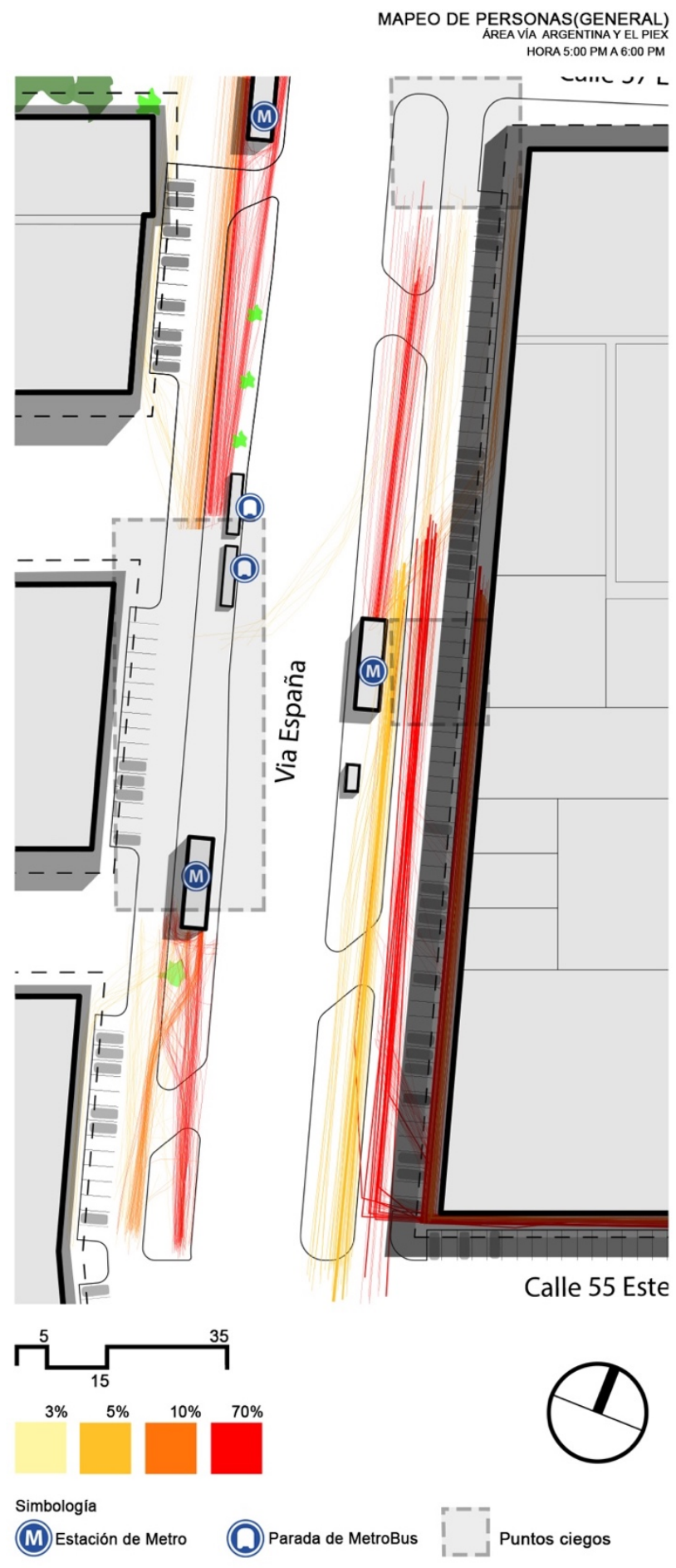

Figura 7. Gráfico General: Flujo de peatones, horario de 5:00 p. m. 6:00 p. m.

Nota: La intensidad de las líneas en los distintos gráficos responde a la cantidad total de peatones en cada uno de los casos. 
Recapitulando el análisis en la PC-1, el promedio fue de 1254 persona en una hora, donde identificamos que el $55 \%$ prefieren transitar por el área abierta y alrededor de $30 \%$ a $55 \%$ por el área techada.

Esto nos indica que a pesar de que el peatón percibe un ambiente fresco en la parte externa, mantiene su preferencia de transitar por el espacio de transición, ya que posee un ambiente más agradable al brindar resguardo. En otras palabras, el espacio de transición no solo es funcional para el usuario en momentos de mayor exposición solar.

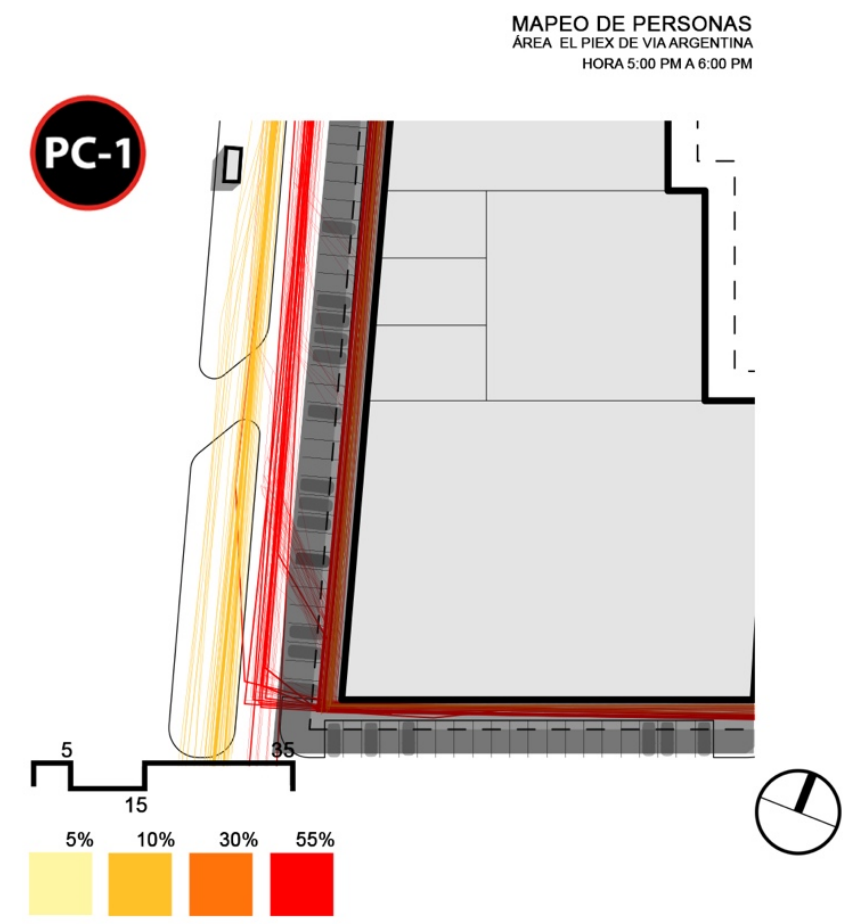

Figura 8. Gráfico de resultado: Ampliación del Piex, horario de 5:00 p. m. 6:00 p. m.

\section{Discusión}

Se analizó más sobre lo que sucede en el área de estudio y la manera en la cual los peatones realizan el desplazamiento en los distintos espacios de transición encontrados. Mediante la elaboración de los resultados, los mapeos de peatones y las encuestas realizadas a los usuarios, logramos entender que el factor principal que afecta en la escogencia del espacio de transición es el clima, ya que influye directamente sobre su confort. Es probable que las sombras producidas por los edificios del sector puedan estar influenciando los patrones al caminar (walking behavior), ya que muchos peatones buscan refugio en las aceras con sombra, exponiendo la importancia de los espacios de transición dentro de la configuración ideal de la ciudad.

Se utilizaron dos métodos para captar los datos (cuantitativos y cualitativos), para así obtener resultados que en conjunto dan más veracidad a la investigación, ya que no fue suficiente con los videos para llegar a los resultados de nuestra investigación.

La mayoría de los peatones transitaban por el área en las horas más elevadas de la tarde debido a que esta responde al promedio de hora de salida de las labores en la ciudad de Panamá y la mayoría de estos utilizan el transporte público (En especial el Metro de Panamá) para realizar la transición entre los espacios.

Estos datos a su vez indicados en los diferentes mapas, muestran que muchos de los peatones, realizan su desplazamiento de tal forma que van cambiando su trayecto entre los espacios de transición y también transitan por espacios que no deberían, por ejemplo, el área de los estacionamientos, olvidando que es un área donde pasan los automóviles. Es posible que esto ocurra como resultado del diseño y la asociación del mismo material de ambos espacios, incorporados desde que se realizó la renovación urbana a lo largo de la Vía España.

También es importante recalcar el motivo del desplazamiento de los peatones, que según los resultados muestra que la mayoría pasan por el área estudiada para llegar a sus lugares de trabajo. Esto es importante ya que el área de estudio tiene a sus alrededores áreas en las cuales hay desarrollo de comercios y oficinas; y nos sugiere que el uso de suelo es un factor que brinda vitalidad al entorno.

En comparación con las demás áreas próximas (Banco Nacional de Panamá, Plaza Regency y Galerías Obarrio), la estación Vía Argentina representa el proyecto de mayor extensión dentro de los nodos, debido a la cantidad de estaciones de transporte público y que posee una configuración distinta. Lo más importante a destacar que es un estudio empírico, que no cuenta con estudios previos.

- Los espacios de transición se ven interrumpidos continuamente y no están bien definidos.

Dentro de las dificultades existentes, y las limitaciones por el equipo, la investigación se ve en la necesidad de dejar áreas sin contabilizar, por lo que se recomienda en futuros trabajos, utilizar cámaras con un campo de visión gran angular de 17.2 mm para lograr un mejor alcance.

\section{Conclusiones}

- Del mapeo general del trayecto de los peatones en el horario de 12:00 m. a 1:00 p. m., el 45\% de los usuarios transitaron por el área abierta próxima a la Vía España, y un 40\% por el área techada; sin embargo, de 5:00 p. m. a 6:00 p. m., el 70\% emplean la acera paralela a la Vía España. Esto es indicador de que la exposición solar afecta directamente en el comportamiento al caminar.

- Del total de usuarios encuestados (40 personas), el $44.8 \%$ se encuentran motivados a transitar por estudios y el $41.4 \%$ por trabajo, es decir que la mayoría realiza una 
caminata utilitaria a comparación de la caminata recreativa.

- Camina por la misma área en la tarde (de 5 a 6 p. m.). Esto es un indicador que el factor de exposición solar afecta directamente sobre el comportamiento al caminar, y la existencia de un espacio de transición mejora el confort del peatón.

- Esta investigación propone la medición de datos cualitativos y cuantitativos, basándonos en una metodología existente que puede utilizarse a futuro en otras áreas con características similares.

\section{AGRADECIMIENTO}

Los autores agradecen al grupo de investigación científica SusBCity por facilitar parte del equipo necesario para realizar las filmaciones y por brindarnos acceso a la plataforma Science Direct administrada por SENACYT.

Los autores agradecen también al Metro de Panamá por facilitarnos datos del uso de la estación Vía Argentina. Este estudio es parte del proyecto de investigación "Diseño sostenible del edificio y su impacto en la ciudad" identificado con código VIP-01-03-01-2016-04 cuyo investigador principal es el Dr. Jorge Isaac Perén Montero.

\section{REFERENCIAS}

[1] R. Rafiemanzelat, M. I. Emadi and A. J. Kamali, "City Sustainability: the influence of walkability on built environments" in 3rd Conference on Sustainable Urban Mobility, Greese, VOL, 2016, pp. 107-104.

[2] B. Kang, A. V. Moudon, P. M. Hurvitz and B. E. Saelens, "Differences in behavior, time, location, and built environment between objectively measured utilitarian and recreational walking", Transportation Research Part D, No. 57, pp. 185194, October 2017.

[3] M. J. Koohsari, H. Badland and B. Giles-Corti, “(Re) Designing the built environment to support physical activity: Bringing public health back into urban design and planning", Cities, No. 35, pp. 294-298, August 2013.

[4] C. Tudor-Locke, M. Bittman and D. Merom, "Patterns of walking for transport and exercise: a novel application of time use data" International Journal of Behavioral Nutrition and Physical Activity, vol. 2, No. 5, May 2005.

[5] R. H. Lo, "Walkability: What is it?" Jorunal of Urbanism: International Research on Placemaking and Urban Sustainability, vol. 2, No. 2, pp. 145-166, July. 2009.

[6] C. Chun, A. Kwok and A. Tamura, "Thermal confort in transitional spaces- basic concepts: literatura review and trial measurement" building and Environment", vol. 39, No. X pp 1187-1192, February 2005.

[7] R. Singh, "Factors affecting walkability of neighborhoods" in Urban Planning and Architecture Design for Sustainable Develop. m.ent, Italy, LE, 2015, pp. 643-654.
- Alrededor del 70\% de los peatones camina por el área techada del Piex al medio día (12 a 1p. m.) y del 30 a 55\%

[8] M. Morales, "Tabla de referencia con detalles de usuarios/hora en la estación vía argentina", Metro de Panamá, Panamá, May 2018. 